\title{
Classification of the Weyl Tensor in Higher Dimensions
}

\author{
A. Coley†, R. Milson†, V. Pravdał, and A. Pravdováł \\ $\dagger$ Department of Mathematics and Statistics, Dalhousie University, Halifax, Nova Scotia \\ $\ddagger$ Mathematical Institute, Academy of Sciences, Žitná 25, 11567 Prague 1, Czech Republic \\ aac@mathstat.dal.ca,milson@mathstat.dal.ca,pravda@math.cas.cz,pravdova@math.cas.cz
}

\begin{abstract}
We discuss the algebraic classification of the Weyl tensor in higher dimensional Lorentzian manifolds. This is done by characterizing algebraically special Weyl tensors by means of the existence of aligned null vectors of various orders of alignment. Further classification is obtained by specifying the alignment type and utilizing the notion of reducibility. For a complete classification it is then necessary to count aligned directions, the dimension of the alignment variety, and the multiplicity of principal directions. The present classification reduces to the classical Petrov classification in four dimensions. Some applications are briefly discussed.
\end{abstract}

[PACS: 04.20.J, 98.90]

The study of higher dimensional manifolds in gravity theory is currently of great interest [1-3]. In particular, finding spacetime models in higher dimensions in the context of string theory, especially in ten and eleven dimensional supergravity theories [4] and branes embedded in higher dimensional bulk manifolds [5], is of fundamental importance. Therefore, a mathematical classification of higher dimensional manifolds is of particular importance. Recently, the algebraic structure of tensors in higher dimensional Lorentian manifolds, including the Riemann and Weyl tensors, has been discussed [6]. Here we shall concentrate on the Weyl tensor and present a higher dimensional algebraic classification which is a generalization of the Petrov classification in four dimensions [7].

We shall consider a null frame $\ell=\mathbf{m}_{\mathbf{0}}, \mathbf{n}=\mathbf{m}_{\mathbf{1}}, \mathbf{m}_{\mathbf{2}}, \ldots \mathbf{m}_{\mathbf{i}}\left(\ell, \mathbf{n}\right.$ null with $\ell^{a} \ell_{a}=n^{a} n_{a}=0, \ell^{a} n_{a}=1, \mathbf{m}^{\mathbf{i}}$ real and spacelike with $m_{i}{ }^{a} m_{j a}=\delta_{i j}$; all other products vanish) in an $N$-dimensional Lorentz-signature space(time), so that $g_{a b}=2 l_{(a} n_{b)}+\delta_{j k} m_{a}^{j} m_{b}^{k}$. Throughout, Roman indices $a, b, c, A, B, C$ range from 0 to $N-1$. Lower case indices indicate an arbitrary basis, while the upper-case ones indicate a null frame. Space-like indices $i, j, k$ also indicate a null-frame, but vary from 2 to $N-1$ only. We will use Einstein's summation convention for both of these types of indices; however, note that for indices $i, j \ldots$ there is no difference between covariant and contravariant components and thus we will not distinguish between subscripts and superscripts.

The frame is covariant relative to the group of linear Lorentz transformations. A null rotation about $\mathbf{n}$ is a Lorentz transformation of the form

$$
\hat{\mathbf{n}}=\mathbf{n}, \quad \hat{\mathbf{m}}_{\mathbf{i}}=\mathbf{m}_{\mathbf{i}}+\mathbf{z}_{\mathbf{i}} \mathbf{n}, \quad \hat{\ell}=\ell-\mathbf{z}_{\mathbf{i}} \mathbf{m}^{\mathbf{i}}-\frac{1}{2} \delta^{\mathbf{i j}} \mathbf{z}_{\mathbf{i}} \mathbf{z}_{\mathbf{j}} \mathbf{n} .
$$

A null rotation about $\ell$ has an analogous form. A boost is a transformation of the form

$$
\hat{\mathbf{n}}=\lambda^{-1} \mathbf{n}, \quad \hat{\mathbf{m}}_{\mathbf{i}}=\mathbf{m}_{\mathbf{i}}, \quad \hat{\ell}=\lambda \ell, \quad \lambda \neq \mathbf{0} .
$$

A spin is a transformation of the form

$$
\hat{\mathbf{n}}=\mathbf{n}, \quad \hat{\mathbf{m}}_{\mathbf{i}}=\mathbf{X}_{\mathbf{i}}^{\mathbf{j}} \mathbf{m}_{\mathbf{j}}, \quad \hat{\ell}=\ell,
$$

where $X_{i}^{j}$ is an orthogonal matrix.

Let $T_{a_{1} \ldots a_{p}}$ be a rank $p$ tensor. For a fixed list of indices $A_{1}, \ldots, A_{p}$, we call the corresponding $T_{A_{1} \ldots A_{p}}$ a null-frame scalar. These scalars transform under a boost (2) according to

$$
\hat{T}_{A_{1} \ldots A_{p}}=\lambda^{b} T_{A_{1} \ldots A_{p}}, \quad b=b_{A_{1}}+\ldots+b_{A_{p}},
$$

where $b_{0}=1, \quad b_{i}=0, \quad b_{1}=-1$. We call the above $b$ the boost-weight of the scalar. We define the boost order of the tensor $\mathbf{T}$ to be the boost weight of its leading term.

We introduce the notation

$$
T_{\{p q r s\}} \equiv \frac{1}{2}\left(T_{[a b][c d]}+T_{[c d][a b]}\right) .
$$

We can decompose the Weyl tensor and sort the components of the Weyl tensor by boost weight [6]: 


$$
\begin{aligned}
& C_{a b c d}=\overbrace{4 C_{0 i 0 j} n_{\{a} m^{i}{ }_{b} n_{c} m_{d\}}^{j}}^{2}+\overbrace{\left.8 C_{010 i} n_{\{a} \ell_{b} n_{c} m_{d\}}^{i}+4 C_{0 i j k} n_{\{a} m^{i}{ }_{b} m^{j}{ }_{c} m^{k}{ }_{d}\right\}}{ }^{1}+ \\
& \left\{\begin{array}{l}
4 C_{0101} n_{\{a} \ell_{b} n_{c} \ell_{d\}}+4 C_{01 i j} n_{\{a} \ell_{b} m^{i}{ }_{c} m^{j}{ }_{d\}}+ \\
8 C_{0 i 1 j} n_{\{a} m^{i}{ }_{b} \ell_{c} m^{j}{ }_{d\}}+C_{i j k l} m_{\{a}^{i} m^{j}{ }_{b} m^{k}{ }_{c} m^{l}{ }_{d\}}
\end{array}\right\}^{0}+ \\
& \overbrace{8 C_{101 i} \ell_{\{a} n_{b} \ell_{c} m_{d\}}^{i}+4 C_{\left.1_{i j k} \ell_{\{a} m_{b}^{i} m^{j}{ }_{c} m^{k} d\right\}}}^{-1}+\overbrace{\left.4 C_{1 i 1 j} \ell_{\{a} m_{b}{ }_{b} \ell_{c} m^{j} d\right\}}^{-2} \text {. }
\end{aligned}
$$

The boost weights for the scalars of the Weyl curvature tensor is given explicitly in (6) (this is summarized concisely in Table I in [6]). The Weyl tensor is generically of boost order 2. If all $C_{0 i 0 j}$ vanish, but some $C_{010 i}$, or $C_{0 i j k}$ do not, then the boost order is 1 , etc. The Weyl scalars also satisfy a number of additional relations, which follow from curvature tensor symmetries and from the trace-free condition:

$$
\begin{gathered}
C_{0 i 0}^{i}=0, C_{010 j}=C_{0 i j}{ }^{i}, C_{0(i j k)}=0, C_{0101}=C_{0 i 1}{ }^{i}, C_{i(j k l)}=0, \\
C_{0 i 1 j}=-\frac{1}{2} C_{i k j}{ }^{k}+\frac{1}{2} C_{01 i j}, C_{011 j}=-C_{1 i j}{ }^{i}, C_{1(i j k)}=0, C_{1 i 1}{ }^{i}=0 .
\end{gathered}
$$

A null rotation about $\ell$ fixes the leading terms of a tensor, while boosts and spins subject the leading terms to an invertible transformation. It follows that the boost order of a tensor is a function of the null direction $\ell$ (only). We shall therefore denote boost order by $\mathcal{B}(\ell)[6]$. We will define a null vector $\ell$ to be aligned with the Weyl tensor whenever $\mathcal{B}(\ell) \leq \mathbf{1}$ (and we shall refer to $\ell$ as a Weyl aligned null direction (WAND)). We will call the integer $1-\mathcal{B}(\ell) \in\{\mathbf{0}, \mathbf{1}, \mathbf{2}, \mathbf{3}\}$ the order of alignment. The alignment equations are $\frac{1}{2} N(N-3)$ degree-4 polynomial equations in $(N-2)$ variables, which are in general overdetermined and hence have no solutions for $N>4$ (i.e., only when these equations are degenerate do we obtain algebraically special spacetimes). Grobner bases can be efficiently used to study this variety and determine any WANDs.

Principal Classification:

Following [6], we will say that the principal type of a Lorentzian manifold is I, II, III, $\mathbf{N}$ according to whether there exists an aligned $\ell$ of alignment order $0,1,2,3$ (i.e. $\mathcal{B}(\ell)=\mathbf{1}, \mathbf{0}, \mathbf{- 1}, \mathbf{- 2}$ ), respectively. If no aligned $\ell$ exists we will say that the manifold is of (general) type $\mathbf{G}$. If the Weyl tensor vanishes, we will say that the manifold is of type O. The algebraically special types are summarized as follows (using (7)):

$$
\begin{aligned}
& \text { Type I: } \quad C_{0 i 0 j}=0 \\
& \text { Type II : } \quad C_{0 i 0 j}=C_{0 i j k}=0 \\
& \text { Type III : } \quad C_{0 i 0 j}=C_{0 i j k}=C_{i j k l}=C_{01 i j}=0 \\
& \text { Type N : } \quad C_{0 i 0 j}=C_{0 i j k}=C_{i j k l}=C_{01 i j}=C_{1 i j k}=0
\end{aligned}
$$

\section{Secondary Classification:}

(i) The existence of aligned null vectors of various orders of alignment can be used to covariantly characterize algebraically special Weyl tensors. Further categorization can be obtained by specifying alignment type [6], whereby we try to normalize the form of the Weyl tensor by choosing both $\ell$ and $\mathbf{n}$ in order to set the maximum number of leading and trailing null frame scalars to zero. Let $\ell$ be a WAND whose order of alignment is as large as possible. We then define the principal (or primary) alignment type of the tensor to be $b_{\max }-b(\ell)$. Supposing such a WAND $\ell$ exists, we then let $\mathbf{n}$ be a null vector of maximal alignment subject to $\ell_{a} n^{a}=1$. We define the secondary alignment type of the tensor to be $b_{\max }-b(\mathbf{n})$. The alignment type of the Weyl tensor is then the pair consisting of the principal and secondary alignment type. The possible alignment types of a higher dimensional Weyl tensor are categorized in Table 4 of [6]. In general, for types $\mathbf{I}, \mathbf{I I}, \mathbf{I I I}$ there does not exist a secondary aligned $\mathbf{n}$ (in contrast to the situation in four dimensions), whence the alignment type consists solely of the principal alignment type. Alignment types $(1,1),(2,1)$ and $(3,1)$ therefore form algebraically special subclasses of types $\mathbf{I}, \mathbf{I I}, \mathbf{I I I}$ respectively (denoted types $\mathbf{I}_{i}, \mathbf{I I}_{i}, \mathbf{I I I}_{i}$ ). There is one final subclass possible, namely type $(2,2)$ which is a further specialization of type $(2,1)$; we shall denote this as type $\mathbf{I I}_{i i}$ or simply as type $\mathbf{D}$. Therefore, a type $\mathbf{D}$ Weyl tensor in canonical form has no terms of boost weights $2,1,-1,-2$ (i.e., all terms are of boost weight zero for type $\mathbf{D}$ ).

(ii) The above classification(s) can be utilized for arbitrary Lorentzian manifolds. In the case in which the Weyl tensor is reducible, it is possible to obtain much more information by decomposing the Weyl tensor and classifying its irreducible parts. For example, we shall say that $C_{a b c d}$ is reducible if there exists a null frame and a constant $M<N$ such that

$$
{ }^{N} C_{a b c d}={ }^{M} C_{a b c d}+\tilde{C}_{\tilde{a} \tilde{b} \tilde{c} \tilde{d}}
$$


where the only non-vanishing components of ${ }^{N} C_{a b c d}$ are

$$
{ }^{M} C_{a b c d} \neq 0, \quad 0 \leq a, b, c, d \leq M-1 ; \quad \tilde{C}_{\tilde{a} \tilde{\tilde{c}} \tilde{d} \tilde{d}} \neq 0, \quad M \leq \tilde{a}, \tilde{b}, \tilde{c}, \tilde{d} \leq N-1 .
$$

That is, $C_{a b c d}$ is (algebraically) reducible if and only if it is the sum of two Weyl tensors. A reducible Weyl tensor is said to be (geometrically) decomposable if and only if the components belonging to $M_{M}$ (resp. $\tilde{M}_{\tilde{M}}$ ) depend on only $x^{c}$ (resp. $y^{\tilde{c}}$ ) [8]: ${ }^{M} C_{a b c d}$ is a Weyl tensor of an (irreducible) Lorentzian spacetime of dimension $M, \tilde{C}_{\tilde{a} \tilde{b} \tilde{c} \tilde{d}}$ is a Weyl tensor of a Riemannian space of dimension $N-M$, and we can write symbolically: $C_{N}=C_{M} \oplus \tilde{C}_{\tilde{M}}$. We can also write a decomposable Weyl tensor in the suggestive block diagonal form: blockdiag $\left\{C_{a b c d}\left(x^{e}\right), \tilde{C}_{\tilde{a} \tilde{b} \tilde{c} \tilde{d}}\left(y^{\tilde{e}}\right)\right\}$. This can be trivially generalized to the case where $\tilde{M}_{\tilde{M}}$ is further reducible. Writing out the Weyl tensor in terms of boost weights we obtain $(6)$, with indices $a, b, c, .$. running from $0-(M-1)$, and an additional term

$$
\text { (+) }\left\{\tilde{C}_{\tilde{i} \tilde{j} \tilde{k} l} m_{\{\tilde{a}}^{\tilde{i}} m_{\tilde{b}}^{\tilde{j}} m^{\tilde{k}} m_{\tilde{c}}^{\tilde{l}}\right\}^{0} .
$$

This term, corresponding to the Riemannian part of the Weyl tensor $\tilde{C}$, is either identically zero (of type $\mathbf{O}$ ) or has terms of boost weight zero only and hence is of type $\mathbf{D}$.

An important auxiliary question is whether we can deduce anything about the manifold structure if the Weyl tensor is reducible. For example, if $M$ is a paracompact, Hausdorff, simply connected smooth Lorentzian manifold, in the case of vacuum it follows that if the Weyl tensor is reducible then it is also decomposable, whence there exists a real non-trivial covariantly geometrical field which is necessarily invariant under the holonomy group (and hence the manifold has a reducible holonomy group). It then follows that the metric in $M$ is the direct product of the metrics in $M_{M}$ and $\tilde{M}_{\tilde{M}}$ and the manifold has a product structure $M_{N}=M_{M} \times \tilde{M}_{\tilde{M}}(N=M+\tilde{M})$, where $M_{M}$ denotes an M-dimensional Lorentzian manifold and $\tilde{M}_{\tilde{M}}$ an $\tilde{M}$-dimensional Riemannian manifold [8].

Two conformally related manifolds have the same Weyl tensor, and therefore their algebraic classifications will be equivalent. In particular, suppose the Lorentzian manifold is an N-dimensional warped product manifold: $M_{N}=$ $M_{M} \times{ }_{F} \tilde{M}_{\tilde{M}}$, with metric $g_{N}=g_{M} \oplus_{F} \tilde{g}_{\tilde{M}}$, where the warp factor $F$ is a function defined on $M_{M}$. This is conformal to the product manifold with metric $\bar{g}_{N}=F\left\{\bar{g}_{M} \oplus \tilde{g}_{\tilde{M}}\right\}$, and hence the warped manifold has the same Weyl classification as the conformally related product manifold. We note that almost all higher dimensional manifolds of physical interest are either product or warped product manifolds [4] (and often are product manifolds obtained by the simple lifting of a lower dimensional spacetime).

Let us assume that the Weyl tensor is reducible as in (9), where ${ }^{M} C$ and $\tilde{C}$ are the $M$ - dimensional irreducible Lorentzian and $N-M$ - dimensional Riemannian parts. Then associated with each part would be a principal type. The principal type of the Lorentzian ${ }^{M} C$ would be $\mathbf{G}, \mathbf{I}, \mathbf{I I}, \mathbf{I I I}, \mathbf{N}, \mathbf{O}$. However, the principal type of the Riemannian $\tilde{C}$ is either $\mathbf{D}$ (type $\mathbf{I I}$, but necessarily all terms of negative boost weight are also zero) or $\mathbf{O}$ (the components of $\tilde{C}$ are identically zero). We denote the secondary type (ii) of a reducible Weyl tensor (9) as $T_{M} \times \tilde{T}_{\tilde{M}}$ or simply by $T_{\tilde{T}}$ if the dimensions $M, \tilde{M}$ are clear. For example, $\mathbf{I}_{\mathbf{D}}$ denotes a reducible Weyl tensor in which the irreducible Lorentzian part of the Weyl tensor is of principal type $\mathbf{I}$, and the irreducible Riemannian part of the Weyl tensor is non-zero. All secondary types are possible, but we note that while $\mathbf{G}_{\mathbf{D}}$ and $\mathbf{G}_{O}, \mathbf{I}_{\mathbf{D}}$ and $\mathbf{I}_{O}, \mathbf{I I}_{\mathbf{D}}$ and $\mathbf{I I}_{O}$ are of principal types $\mathbf{G}, \mathbf{I}, \mathbf{I I}$, respectively, only types $\mathbf{I I I}_{\mathbf{O}}, \mathbf{N}_{\mathbf{O}}, \mathbf{O}_{\mathbf{O}}$ are of principal types $\mathbf{I I I}, \mathbf{N}, \mathbf{O}$ (resp.); secondary types $\mathbf{I I I}_{\mathbf{D}}, \mathbf{N}_{\mathbf{D}}, \mathbf{O}_{\mathbf{D}}$ are all of principal type $\mathbf{I I}$.

The secondary types of the form (i) and (ii) can of course be combined with the obvious (albeit somewhat clumsy) notation. Note that although all of the secondary type (ii)s $\mathbf{I I}_{\mathbf{D}}, \mathbf{I I I}_{\mathbf{D}}, \mathbf{N}_{\mathbf{D}}, \mathbf{O}_{\mathbf{D}}$ are of principal type $\mathbf{I I}$ and the secondary type (i) $\mathbf{I I}_{i i}$ is possible, only secondary type $\mathbf{I I}_{\mathbf{D}, i i}$ is possible (while secondary types $\mathbf{I I I}_{\mathbf{D}, i i}, \mathbf{N}_{\mathbf{D}, i i}, \mathbf{O}_{\mathbf{D}, i i}$ are not possible).

\section{Full Classification:}

Alignment type by itself is insufficient for a complete classification of the Weyl tensor. It is necessary to count aligned directions, the dimension of the alignment variety, and the multiplicity of principle directions. We note that unlike in the four dimensional case, it is possible to have an infinity of aligned directions. If a WAND is discrete, for consistency with four dimensional nomenaclature we shall refer to it as a principal null direction (PND). We can introduce extra normalizations and obtain further subclasses. The classification of higher-dimensional Weyl tensors is made more straightforward by using the notion of reducibility, since we only need classify each irreducible part (the classification of the irreducible Lorentzian piece is discussed here and in [6], and the classification of the Riemannian piece, which is simpler, is discussed in [6] and [9]). However, further complications in attempting to find canonical forms arise due to gauge fixing (i.e., certain terms can be chosen to be zero by an appropriate choice of frame through boosts and spatial rotations).

The full type of an irreducible Weyl tensor $C_{a b c d}$ is defined by its principal and secondary types, and includes all of the information on subcases and multiplicities. We do not classify these in full detail here (indeed, it may 
be necessary to consider different specific dimensions on a case by case basis), but rather describe some of the key algebraically special subtypes below.

First, there are additional conditions for algebraic specializations: (i) in Type I (a) $C_{010 i}=0$, (ii) in Type II (a) $C_{0101}=0$, (b) the traceless Ricci part of $C_{i j k l}=0$, (c) the Weyl part of $C_{i j k l}=0$, (d) $C_{01 i j}=0$, (iii) Type III (a) $C_{011 i}=0$. For example, there are consequently two subcases of Type III, namely type III (general type) and type III (a) in which $C_{011 i}=0$ (see below). Second, there are further specializations due to multiplicities. In types III and $\mathbf{N}$ all WANDs are necessarily PND. For type III tensors, the PND of order 2 is unique. There are no PNDs of order 1 , and at most 1 PND of order 0 . For $N=4$ there is always exactly 1 PND of order 0 . For $N>4$ this PND need not exist. For type $\mathbf{N}$ tensors, the order 3 PND is the only PND of any order.

We can write a canonical form for Weyl type N. From (6), (7) and (8) we have that for type N:

$$
C_{a b c d}=4 C_{1 i 1 j} \ell_{\{a} m_{b}^{i} \ell_{c} m^{j}{ }_{d\}} ; \quad C_{1 i 1}{ }^{i}=0 .
$$

The general form of the Weyl tensor for type III is given by (6) subject to (7) and (8). In the subclass III $_{i}$ we have that $C_{1 i 1 j}=0$ and hence

$$
C_{a b c d}=8 C_{101 i} \ell_{\{a} n_{b} \ell_{c} m^{i}{ }_{d\}}+4 C_{1 i j k} \ell_{\{a} m^{i}{ }_{b} m^{j}{ }_{c} m^{k}{ }_{d\}},
$$

where $C_{011 j}=-C_{1 i j}{ }^{i}, C_{1(i j k)}=0$, and not all of $C_{1 i j k}$ are zero (else it reduces to Weyl type $\mathbf{O}$ ). In the subclass III(a) we have that $C_{011 j}=0$, so that

$$
C_{a b c d}=4 C_{1_{i j k}} \ell_{\{a} m_{b}^{i} m^{j}{ }_{c} m^{k}{ }_{d\}}+4 C_{1 i 1 j} \ell_{\{a} m_{b}^{i} \ell_{c} m^{j}{ }_{d\}},
$$

where $C_{1 i j}{ }^{i}=C_{1(i j k)}=C_{1 i 1}{ }^{i}=0$. There is a further subcase obtained by combining the two cases above (essentially setting the final term in the last equation to zero).

The complete classification for $N=4$ is relatively straightforward, due to the facts that there always exists at least one aligned direction, that all such aligned directions are discrete, normalization reduces the possible number of subclasses (leading to unique subcases) and since reducibility is not an issue because a Weyl tensor defined over a vector space of dimension 3 or less must necessarily vanish. In [6] it was shown that the present classification reduces to the classical Petrov classification in four dimensions.

It is fortunate that in most applications [1-5] the Weyl classification is relatively simple and the details of the more complete classification are not necessary. In the future it would be useful to be able to find a more practical way of determining the Weyl type, such as for example employing certain scalar higher dimensional invariants. We also note that it may be more practical in some situations to classify the Riemann tensor (see [10] for comments on the classification of the Riemann tensor in four dimensions) since it is not considerably more difficult to classify the Riemann tensor rather than the Weyl tensor in higher dimensions [6].

There are many applications of this classification scheme. Recently we have investigated $N$-dimensional Lorentzian spacetimes in which all of the scalar invariants constructed from the Riemann tensor and its covariant derivatives are zero (thereby generalizing the theorem of [11] to higher dimensions). These spacetimes are referred to as vanishing scalar invariant (VSI) spacetimes, and they can be regarded as higher-dimensional generalizations of $N$-dimensional pp-wave spacetimes (which are of interest in the context of string theory in curved backgrounds). In [12] we proved that the higher dimensional VSI spacetimes are necessarily of Weyl type III, $\mathbf{N}$ or $\mathbf{O}$ (and we presented a canonical form for the Riemann and Weyl tensors in a preferred null tetrad in arbitrary dimensions). For algebraically special vacuum type III and $\mathbf{N}$ spacetimes in arbitrary dimensions we have proven that the null congruence is geodesic and shear-free [13]. Further progress towards a generalization of the Goldberg-Sachs theorem is possible in higher dimensions. In [13] we also showed that the Weyl tensor in vacuum type $\mathbf{N}$ spacetimes with non-vanishing expansion or twist is always reducible, with a nontrivial four-dimensional Lorentz part and a vanishing $N-4$ dimensional Euclidean part.

In future work we shall present the details of the full algebraic classification of higher dimensional Weyl tensors. In particular, we shall study the five dimensional case in detail (and relate this classification to previous work [14]), and study type $\mathbf{D}$ spacetimes and look for exact solutions that are generalizations of particular type $\mathbf{D}$ solutions in four dimensions of special interest (these include generalizations of the Schwarzschild solution in higher dimensions, which we note are warped product manifolds). For example, the higher dimensional spherically symmetric vacuum nonrotating black hole solutions [3], which are generalizations of the exact Schwarzschild solution in four dimensions, are of type $\mathbf{D}$, while the five dimensional Sorkin-Gross-Perry-Davidson-Owen soliton solutions [2] are all of (algebraically special) type $\mathbf{I}$, except for the special case of a generalized Schwarzschild black hole solution which is again of type $\mathbf{D}$.

Acknowledgements. We would like to thank Nicos Pelavas for helpful comments. AP and VP would like to thank Dalhousie University for its hospitality while this work was carried out. AC and RM were supported, in part, by a research grant from NSERC. VP was supported by GACR-202/03/P017. 
[1] J.M. Overduin and P.S. Wesson, 1997, Phys. Reports 283, 303; P.S. Wesson, 1999, Space, Time and Matter (World Scientific, Singapore).

[2] R.D. Sorkin, 1983, Phys. Rev. Lett. 51, 87; D.J. Gross and M.J. Perry, 1983, Nucl. Phys. B 226, 29; A. Davidson and D.A. Owen, 1985, Phys. Lett. B 155, 247.

[3] F.R. Tangherlini, 1963, Nuovo Cim. 77, 636: R.C. Myers and M.J.Perry, 1983, Nucl. Phys. B 226, 29

[4] A.A. Tseytlin, 1995, Class. Quant. Grav. 12, 2365; G.H. Horowitz and A.A. Tseytlin, 1995, Phys. Rev D 51, 2896; J.G. Russo and A.A. Tseytlin, 1995, Nucl. Phys. B 448, 293; ibid hep-th/0208114; M. Blau, J. Figueroa-O'Farrill, C. Hull and G. Papadopoulos, 2002, JHEP 0201, 047; ibid. hep-th/0202111; J. Kowalski-Glikman, 1985, Phys. Lett. B 150 , 125.

[5] V. Rubakov and M. Shaposhnikov, 1983, Phys. Lett. B 125, 139; N. Arkani-Hamed, S. Dimopoulos and G. Dvali, 1998, Phys. Lett. B 429, 263; L. Randall and R. Sundrum, 1999, Phys. Rev. Lett. 83, 3370 \& 4690.

[6] R. Milson, A. Coley, V. Pravda, A. Pravdova, 2003, Algebraically Special Tensors, sub. J. Geom. Phys [gr-qc/0401010].

[7] A. Z. Petrov, 1954, Sci. Notices of Kazan State University 114, 55; ibid., 1969, Einstein Spaces (Pergamon Press); R. Penrose and W. Rindler, 1986, Spinors and Space-time, Vol. 2 (Cambridge University Press, Cambridge).

[8] S. Kobayashi and K. Nomizu, 1963, Foundations of Differential Geometry and its Applications, Vol. 1 (Interscience, New York.); B. O'Neill, 1981, Semi-Riemann Manifold (Wiley, New York); J.K. Beem and P.E., Ehrlich, 1981 Global Lorentzian Geometry, (Dekker, New York).

[9] A. Karlhede, 1986, Class. Quantum Grav. 3, L1.

[10] B.H. Haddow, gr-qc/9507007; S.A. Fulling, R.C. King, B.G. Wybourne and C.J. Cummins, 1992, Class. Quantum Grav. 9, 1151.

[11] V. Pravda, A. Pravdová, A. Coley and R. Milson, 2002, Class. Quant. Grav. 12, 2365.

[12] A. Coley, R. Milson, N. Pelavas, V. Pravda, A. Pravdova and R. Zalaletdinov, 2002, Phys. Rev. D. 67, 104020; A. Coley, R. Milson, V. Pravda, A. Pravdova, 2003, Higher Dimensional VSI spacetimes, sub. Class. Quant. Grav.

[13] V. Pravda, A. Pravdová, A. Coley and R. Milson, 2003, Biachi Identities in Higher Dimensions, sub. Class. Quant. Grav. [gr-qc/0401013].

[14] P-J. De Smet, 2003, Class. Quant. Grav. 20, 2541; see also gr-qc/0306026. 